\title{
Article \\ A Model for Dose Dependence of the Void Swelling in Electron-Irradiated Alloys
}

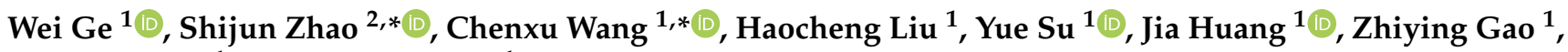 \\ Jianming Xue ${ }^{1}$ and Yugang Wang ${ }^{1}$
}

1 State Key Laboratory of Nuclear Physics and Technology, Center for Applied Physics and Technology, Peking University, Beijing 100871, China; gewei@pku.edu.cn (W.G.); liuhaocheng@pku.edu.cn (H.L.); yuesu@pku.edu.cn (Y.S.); jiahuang@pku.edu.cn (J.H.); zhiyinggao@pku.edu.cn (Z.G.); jmxue@pku.edu.cn (J.X.); ygwang@pku.edu.cn (Y.W.)

2 Department of Mechanical Engineering, City University of Hong Kong, Hong Kong, China

* Correspondence: shijzhao@cityu.edu.hk (S.Z.); cxwang@pku.edu.cn (C.W.)

Citation: Ge, W.; Zhao, S.; Wang, C.; Liu, H.; Su, Y.; Huang, J.; Gao, Z.; Xue, J.; Wang, Y. A Model for Dose Dependence of the Void Swelling in Electron-Irradiated Alloys. Metals 2022, 12, 244. https://doi.org/ $10.3390 /$ met12020244

Academic Editors: Lingfeng He and Hannu Hänninen

Received: 1 December 2021

Accepted: 25 January 2022

Published: 27 January 2022

Publisher's Note: MDPI stays neutral with regard to jurisdictional claims in published maps and institutional affiliations.

Copyright: (c) 2022 by the authors. Licensee MDPI, Basel, Switzerland. This article is an open access article distributed under the terms and conditions of the Creative Commons Attribution (CC BY) license (https:// creativecommons.org/licenses/by/ $4.0 /)$.

\begin{abstract}
Understanding the void swelling dependence on irradiation dose for structural materials is critical for the design and operation of advanced nuclear reactors. Due to their easy accessibility in high-voltage transmission electron microscopes, electron beams have been frequently employed to investigate the void swelling mechanisms. Here, we build a general model to describe the radiation-induced swelling produced by energetic electrons. Based on this model, we develop a quantitative relation between void swelling and irradiation dose, which is in good agreement with experimental data. By extrapolating to high-dose swelling in electron-irradiated alloys, our model validation is consistent with available experiments. Furthermore, the model is well supported by our phase-field simulations.
\end{abstract}

Keywords: structural alloys; electron irradiation; radiation-induced void swelling; dose dependence

\section{Introduction}

The development of advanced nuclear energy systems is one of the most promising ways to address the anticipated global climate change and energy crisis. One of the major problems for the development of structural materials in advanced nuclear energy systems is the swelling induced by neutron radiation-induced void growth [1,2], which has been widely investigated since the 1960s [2,3]. Void swelling is a result of the evolution of radiation-induced defects. It is well established that energetic particles interact with materials by transferring their kinetic energies into the electronic and atomic subsystems of target materials. This process can be described by two main simplified processes [4]: (1) energetic particles first collide with lattice atoms and produce primary knock-on atoms (PKAs), giving rise to Frenkel defects with the same overall number of interstitials and vacancies. If the PKAs have sufficient kinetic energy, they can lead to further atomic cascade displacements and produce more point defects and defect clusters. (2) Defects evolution occurs via either recombination of interstitials and vacancies or diffusion-induced defect absorption/accumulation at various defect sinks, such as dislocations, defect clusters, grain boundaries, interfaces, and precipitates. The biased absorption for point defects by the biased sinks leaves excess point defects during irradiation [5]. The remaining mobile vacancies aggregate for void formation and growth.

To compress the time scale required for studying this problem, many researchers have employed electron beams to study the radiation-induced void swelling in alloys [6]. Since the 1970s, the energetic electron beam in the high voltage electron microscope (HVEM) has been extensively used for irradiation and swelling investigation [4,7-11]. In HVEM, electron beam for irradiation can be used to simultaneously image the damage. Meanwhile, the high dose rate of electron irradiation in HVEM (3-4 orders of magnitude higher than 
that under nuclear reactor neutron irradiation) allows for a very short irradiation time [12] (several hours) to achieve a high irradiation dose that otherwise requires several years of neutron irradiation in nuclear reactors. Most importantly, in HVEM, the energies of electrons are in the order of $1 \mathrm{MeV}$, which are sufficient for producing isolated Frenkel pairs which are uniformly distributed in the specimen, but not collision cascades $[13,14]$. Thus, electron irradiation has been of the foremost importance in the understanding of radiation-induced void swelling effects and the development of void swelling models based on diffusion-controlled void growth of point defects.

There are wide disagreements concerning the functional relationship between swelling and dose, the same set of experimental data may be fitted by different empirical equations [14], and different sets of experimental data can be fitted by different empirical equations. For example, the result in some experiments shows that the dose dependence of void swelling is (dose) ${ }^{1.58}$ in HVEM [6], where the dose is in the unit of dpa (displacement per atom [15]). Nonetheless, the best fitting exponents of other experiments may be 1.1, $1.5,1.8$ or 2.2 [14]. It was even claimed that a linear relationship between the swelling and the irradiation dose is expected at high swelling levels [4]. Particularly, it is often difficult to evaluate the swelling trend as it is an intrinsically non-linear relationship from low to intermediate doses, swelling in materials exhibits several distinct stages, such as a nucleation/incubation stage, a transient stage, and a steady-growth stage. Until now, there has been no suitable general model to describe the void swelling at different doses in alloys under electron irradiation. Here, we will build a model for dose dependence of the void swelling in electron-irradiated alloys.

\section{Materials and Methods}

Figure 1 shows the radiation damage process produced by energetic electrons, specifically the void formation. The incident energetic electrons generate uniformly distributed Frenkel defects (interstitials and vacancies) without collision cascades. The defects can be either recombined or absorbed by various defect sinks, including dislocations and boundaries. The aggregation of surviving defects leads to void formation and growth.

The governing equations for interstitials and vacancies of above process can by described by defect reaction rate theory, respectively [16],

$$
\begin{gathered}
\frac{\partial c_{i}}{\partial \tau}=G_{i}-R_{i v} c_{i} c_{v}-S_{i} c_{i} \\
\frac{\partial c_{v}}{\partial \tau}=G_{v}-R_{i v} c_{i} c_{v}-S_{v} c_{v}
\end{gathered}
$$

where $c_{i}$ and $c_{v}$ are the concentration of interstitials and vacancies, respectively, and $R_{i v}$ is the recombination rate of interstitials and vacancies. Moreover, the bias for the total sink absorption rate $\left(S_{i, v}\right)$ can be expressed as $b=\frac{S_{i}}{S_{v}}$, with the assumption that $S_{i}$ and $S_{v}$ are constant. Since the electron-irradiation induced defects are generated in the form of Frenkel pairs, the generation rate of interstitials $G_{i}$ and the generation rate of vacancies $G_{v}$ are the same, $G_{i}=G_{v}=G$. Suppose local equilibrium is reached ( $\tau$ is sufficiently large), meaning steady-state conditions, the solutions of Equations (1) and (2) give the defect concentrations:

$$
c_{i}=\frac{S_{v}}{S_{i}} c_{v} \text { and } c_{v}=\frac{2 G}{S_{v}+\sqrt{4 G R_{i v} \frac{S_{v}}{S_{i}}+S_{v}^{2}}}
$$

The steady-state solutions are used in the following void swelling model since it is generally considered that defects can achieve their equilibrated states quickly compared to the time scale of void growth [4]. The classical rate theory for void growth is [4]:

$$
\frac{d r}{d t}=\frac{\Omega}{r}\left[D_{v} c_{v}-D_{i} c_{i}\right]
$$


where $r$ is the void radius, $\Omega$ is the defect volume, and $D_{v, i}$ is the diffusion coefficient of vacancies/interstitials. Following previous studies [5], we have omitted the concentrations of thermal vacancies in the void surface. After inserting the solutions of $c_{v, i}$, we have:

$$
\frac{d r}{d t}=\frac{\Omega}{r}\left(D_{v} S_{i}-D_{i} S_{v}\right) \frac{\sqrt{1+\frac{4 G R_{i v}}{S_{i} S_{v}}}-1}{2 R_{i v}}
$$

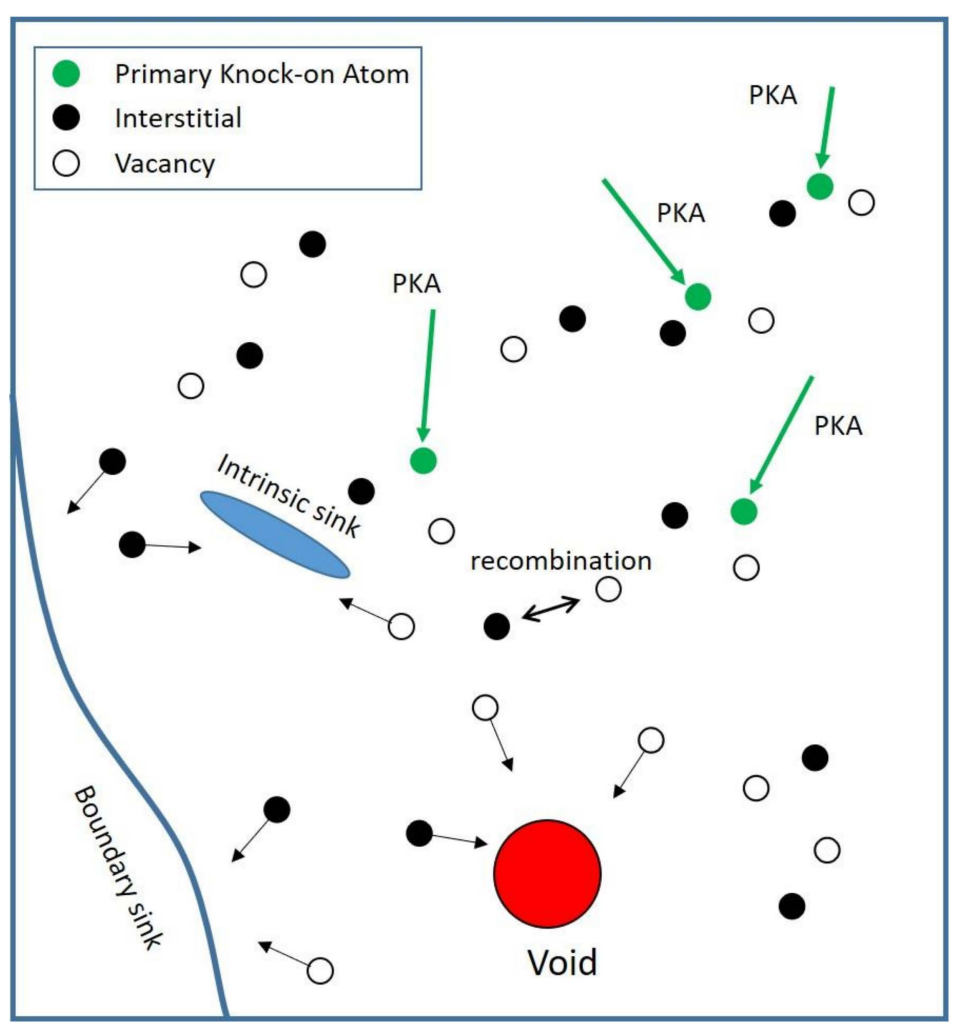

Figure 1. Schematic illustration of electron irradiation effects in materials; PKA-primary knock-on atom.

Since the total defect sinks play majority roles in alloys, the sink dominant case can be considered, i.e., $\frac{4 G R_{i v}}{S_{i} S_{v}} \ll 1$. Therefore, Equation (5) can be simplified to:

$$
\frac{d r}{d t}=\frac{\Omega}{r}\left(D_{v} S_{i}-D_{i} S_{v}\right) \frac{G}{S_{i} S_{v}}
$$

The solution of this equation is $r=\left\{t \frac{2 G \Omega}{S_{v}}\left(D_{v}-\frac{D_{i}}{b}\right)\right\}^{\frac{1}{2}}$. Considering the relations among dose $\left(\Delta_{d p a, \text { ele }}, \mathrm{dpa}\right)$, dose rate $\left(G_{d p a, \text { ele }}, \mathrm{dpa} / \mathrm{s}\right)$, and defect generation rate $\left(G, \mathrm{~m}^{-3} \mathrm{~s}^{-1}\right)$ of electrons, $G_{d p a, e l e}=\eta G$, and $\Delta_{d p a, e l e}=G_{d p a, e l e}$ t, where $\eta$ is the constant ratio between electron irradiation dose rate and defect generation rate, we can rewrite the void radius to:

$$
r=\left\{2 \Omega\left(\frac{D_{v} b-D_{i}}{b S_{v}}\right) \frac{\Delta_{d p a, e l e}}{\eta}\right\}^{\frac{1}{2}}
$$

The volume swelling's relation with irradiation dose is:

$$
\text { swelling }=\frac{\Delta V}{V_{0}}=\frac{\rho_{N} V_{0} \frac{4}{3} \pi r^{3}-\rho_{N} V_{0} \frac{4}{3} \pi r_{0}^{3}}{V_{0}}=\frac{4 \pi \rho_{N}}{3}\left\{2 \Omega\left(\frac{D_{v} b-D_{i}}{b S_{v}}\right) \frac{\Delta_{d p a, e l e}}{\eta}\right\}^{\frac{3}{2}}-\frac{4 \pi \rho_{N} r_{0}^{3}}{3},
$$


where $V_{0}$ is the original volume without voids, $\rho_{N}$ is the number density of voids. We also introduce $r_{0}$ to account for the void nucleation stage. Specifically, the value of $r_{0}$ is the critical size of a void embryo that must be achieved in order for the embryo to grow into a void. The relation between void swelling and irradiation dose becomes:

$$
\text { swelling }=\frac{4 \pi \rho_{N}}{3}\left\{2 \Omega\left(\frac{D_{v} S_{i}-D_{i} S_{v}}{\eta S_{v} S_{i}}\right) \Delta_{d p a, e l e}\right\}^{\frac{3}{2}}-\frac{4 \pi \rho_{N} r_{0}^{3}}{3}=\alpha\left[\Delta_{d p a, e l e}\right]^{\frac{3}{2}}-c \text {, }
$$

where $r_{0}$ is nucleation radius, $\alpha=\frac{4 \pi \rho_{N}}{3}\left[2 \Omega \frac{D_{v} S_{i}-D_{i} S_{v}}{\eta S_{i} S_{v}}\right]^{\frac{3}{2}}, c=\frac{4 \pi \rho_{N} r_{0}^{3}}{3}$.

Equation (9) reveals that void swelling is controlled by material properties and irradiation conditions, where materials properties are combined into parameter $\alpha$ and $c$, irradiation conditions are combined into parameter dose $\left(\Delta_{d p a, e l e}\right.$, in the unit of dpa). Besides, it indicates that swelling is proportional to 1.5 power of irradiation dose.

In summary, the model above provides the quantitative dependence of void swelling on different parameters at a fixed temperature, including material properties ( $\alpha$ and $c)$, irradiation dose. For material properties, $\alpha$ is governed by intrinsic properties such as defect diffusion coefficients and the absorption rate of intrinsic defect sinks, including the influence of temperature, pressure, concentration of the intrinsic sinks [17], and cold-work conditions on these properties. Both the material fabrication and the absorption rate of intrinsic defect sinks affect material parameter $\alpha$ by affecting parameters $S_{i}$ or $S_{v}$. The material parameter $c$ is governed by the void nucleation ability of materials.

\section{Results}

In Equation (9), the separated material parameters and irradiation parameters make validating the functional relationship between swelling and dose of the experiments at the same temperature for the same alloy possible. As the energy for electron irradiation is limited to $1 \mathrm{MeV}$ [12], we can only find low dose and intermediate dose data for electron irradiation, thus, we just apply our model to these experiment results. As shown in Figure 2, the void swelling behavior can be well fitted by our model for different kinds of electronirradiated alloys, where the fitting coefficients of determination (r-squares) are all greater than $97 \%$, as shown in Table 1, where r-square is a statistical measure of how close the data are to the fitted regression line. Void swelling starts to continuously grow once the irradiation dose reaches a threshold value. This can be explained by the nucleation process or incubation period for void swelling that is frequently observed in experiments [18,19]. By extrapolating to the dose where the swelling equals to zero, we obtain the threshold dose $\Delta_{0}$ after which pronounced swelling begins (indicated by an arrow in Figure 2a):

$$
\Delta_{0}=\frac{r_{0}^{2}}{2 \Omega} \frac{\eta S_{i} S_{v}}{D_{v} S_{i}-D_{i} S_{v}}
$$

which reasonably relates to $r_{0}$ and other material parameters. This model thus provides a general description of the radiation-induced swelling behavior over the whole dose range.

In Figure 2, all the irradiation experiments were conducted at a fixed dose rate and temperature for a specific alloy. According to Equation (9), there are only two parameters in the relationship between the swelling and dose, i.e., $\alpha$ and $c$. The obtained parameters are provided in the Table 1 . It is found that $c$ is small in all cases, suggesting a short + for void swelling in electron irradiation.

Suppose that material parameters $(\alpha$ and $c$ ) remain unchanged when it comes to highdose irradiation. Equation (9) can also serve as the basis for extrapolating high swelling level for an identical material at the same temperature and fixed irradiation dose rate from low-dose condition. In each subgraph of Figure 3, 316 SS was irradiated in the same HVEM at the same temperature. The parameters $\alpha$ and $c$ were calculated from the low-dose irradiation data (blue square symbols) [14] which are listed in Table 2. Equation (9) can be used to extrapolate the swelling rate at higher doses. Our extrapolations are consistent with 
another set of high-dose irradiation at the same HVEM (black circle symbols) [14]. Therefore, these examples suggest that our derived model can also be validated by extrapolating void swelling at high doses from the low dose data for electron irradiation.

(a)

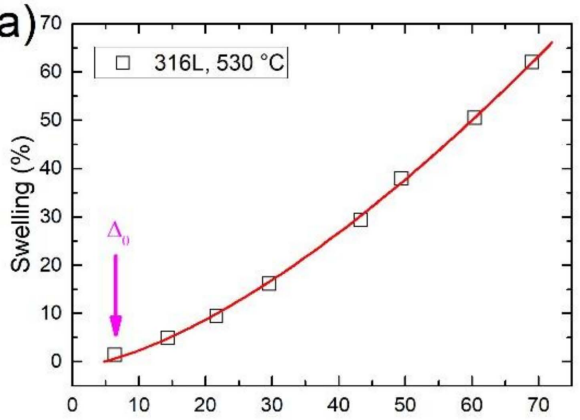

(d)

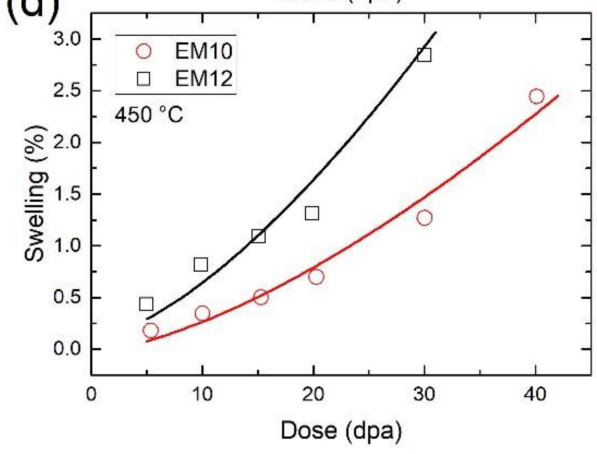

(b)

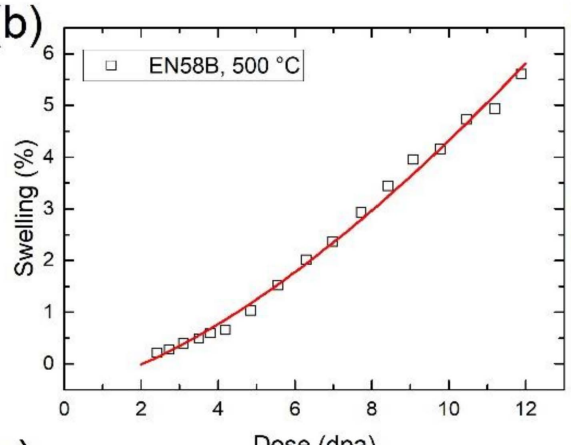

(e)

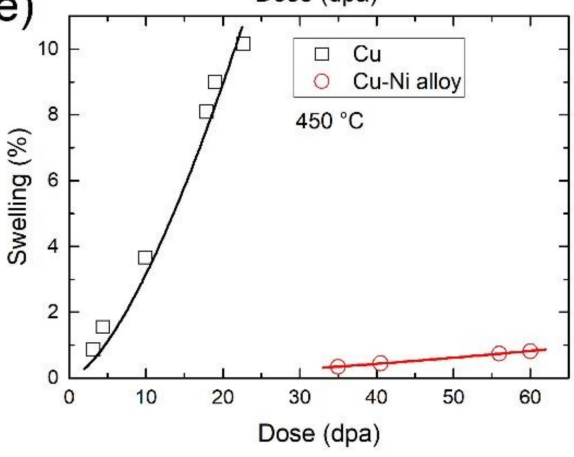

(c)

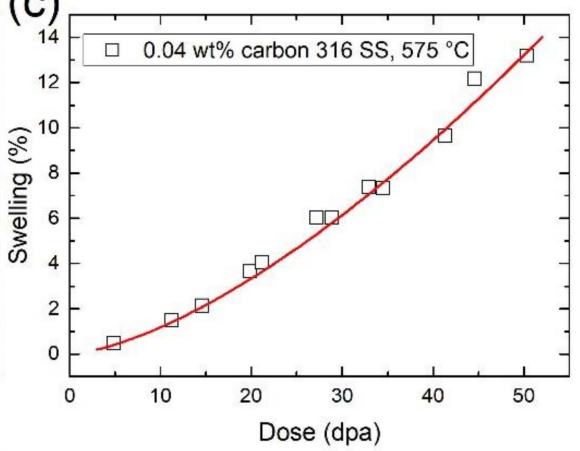

Figure 2. Validation of the model against void swelling data from electron irradiation experiments. (a) 316L alloy irradiated by electrons at $530{ }^{\circ} \mathrm{C}$ [6], (b) EN58B alloy irradiated by electrons at $500{ }^{\circ} \mathrm{C}$ [20], (c) $0.04 \mathrm{wt} \%$ carbon $316 \mathrm{SS}$ (stainless steel) alloy irradiated by electrons at $575{ }^{\circ} \mathrm{C}$ [21], (d) EM10 and EM12 alloys irradiated by electrons at $450{ }^{\circ} \mathrm{C}$ [22], (e) $\mathrm{Cu}$ and $\mathrm{Cu}-\mathrm{Ni}$ alloy irradiated by electrons at $450{ }^{\circ} \mathrm{C}[23]$. The solid lines are our model-fitting results.

Table 1. Fitting parameters of different materials under $1 \mathrm{MeV}$ electron irradiation at different temperatures in Figure 2.

\begin{tabular}{cccc}
\hline Materials & $\alpha$ & $c$ & r-Square \\
\hline 316L, $530{ }^{\circ} \mathrm{C}$ & 0.11008 & 1.16224 & 0.99926 \\
$\mathrm{EN} 58 \mathrm{~B}, 500^{\circ} \mathrm{C}$ & 0.1502 & 0.43211 & 0.99417 \\
$316 \mathrm{SS}, 575{ }^{\circ} \mathrm{C}$ & 0.03737 & 0.00001 & 0.9872 \\
$\mathrm{EM} 10,450{ }^{\circ} \mathrm{C}$ & 0.00908 & 0.02249 & 0.97391 \\
$\mathrm{EM} 12,450{ }^{\circ} \mathrm{C}$ & 0.01812 & 0.00001 & 0.99472 \\
$\mathrm{Cu}, 450{ }^{\circ} \mathrm{C}$ & 0.09994 & 0.00001 & 0.97601 \\
$\mathrm{Cu}-\mathrm{Ni}$ alloy, $450^{\circ} \mathrm{C}$ & 0.00185 & 0.03309 & 0.99909 \\
\hline
\end{tabular}

Table 2. Extrapolation parameters of 316 stainless steel under $1 \mathrm{MeV}$ electron irradiation at different temperatures.

\begin{tabular}{ccc}
\hline Temperature & $\alpha$ & $c$ \\
\hline $500^{\circ} \mathrm{C}$ & 0.01624 & $1.00 \times 10^{-3}$ \\
$550^{\circ} \mathrm{C}$ & 0.02114 & 0.07978 \\
$570^{\circ} \mathrm{C}$ & 0.03273 & 0.62573 \\
$600^{\circ} \mathrm{C}$ & 0.03379 & 1.61698 \\
\hline
\end{tabular}




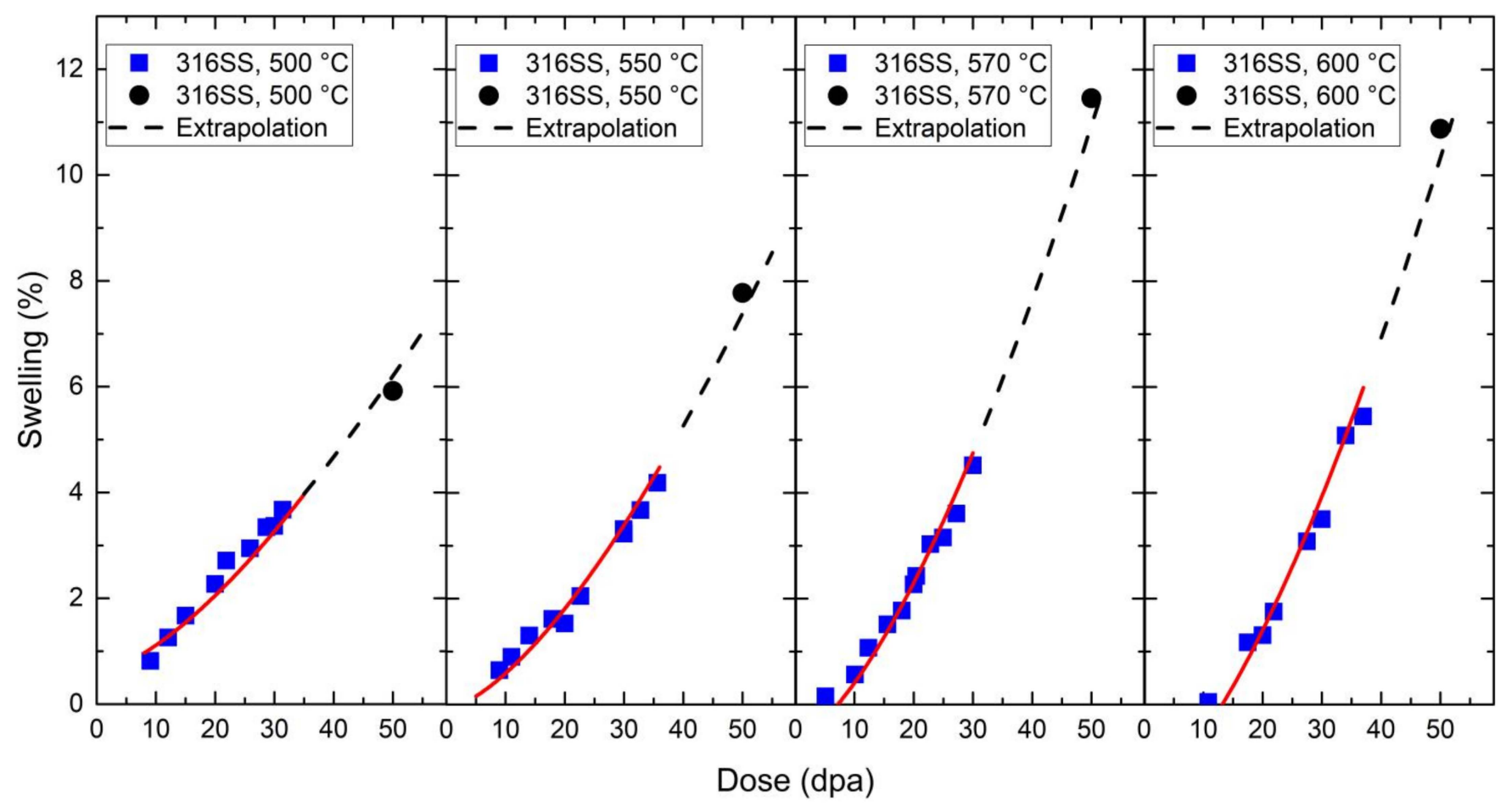

Figure 3. Extrapolations of swelling from low-dose to high-dose electron irradiation. Electron irradiations are conducted at different temperature in the same HVEM for the same material. Each black dash line indicates the extrapolation from parameters that are calculated from a set of irradiation data [14] at low-dose irradiation (blue square symbols) in each subgraph.

\section{Discussion}

In order to further validate the relation derived in Equation (9), we adopted the phase-field model (PFM) of void swelling described by Li [24] and Chang [25]:

$$
\begin{gathered}
\frac{\partial c_{i}}{\partial t}=M_{i} \nabla^{2}\left(\frac{\delta F}{\delta c_{i}}\right)+G_{i}-R_{i v} c_{i} c_{v}-S_{i} c_{i} \\
\frac{\partial c_{v}}{\partial t}=M_{v} \nabla^{2}\left(\frac{\delta F}{\delta c_{v}}\right)+G_{v}-R_{i v} c_{i} c_{v}-S_{v} c_{v},
\end{gathered}
$$

where $M_{i}$ and $M_{v}$ are interstitial mobility and vacancy mobility, $F$ is the total free energy of the system. The total free energy of the system, including the chemical free energy and gradient energy, can be expressed as a function of $c_{i}$ and $c_{v}$ [24]:

$$
F=\int_{V}\left[f\left(c_{i}, c_{v}\right)+\frac{\lambda_{i}}{2}\left(\nabla c_{i}\right)^{2}+\frac{\lambda_{v}}{2}\left(\nabla c_{v}\right)^{2}\right] d V,
$$

where $\lambda_{i}$ and $\lambda_{v}$ are gradient energy coefficients for interstitial and vacancy, respectively. The chemical/bulk energy density $f\left(c_{i}, c_{v}\right)$ can be represented by a widely-used phenomenological double-well potential [26]:

$$
f\left(c_{i}, c_{v}\right)=f\left(c_{i}\right)+f\left(c_{v}\right)=A_{i} c_{i}^{2}\left(1-c_{i}\right)^{2}+A_{v} c_{v}^{2}\left(1-c_{v}\right)^{2},
$$

where $A_{i}$ and $A_{v}$ are positive constants and controls the magnitude of the energy barrier between two equilibrium phases. Therefore, $\frac{\delta F}{\delta c_{i}}$ and $\frac{\delta F}{\delta c_{i}}$ can be derived as:

$$
\frac{\delta F}{\delta c_{i}}=\frac{\delta f}{\delta c_{i}}-\lambda_{i} \nabla^{2} c_{i}=2 A_{i} c_{i}\left(1-c_{i}\right)\left(1-2 c_{i}\right)-\lambda_{i} \nabla^{2} c_{i}
$$




$$
\frac{\delta F}{\delta c_{v}}=\frac{\delta f}{\delta c_{v}}-\lambda_{i} \nabla^{2} c_{v}=2 A_{v} c_{v}\left(1-c_{v}\right)\left(1-2 c_{v}\right)-\lambda_{i} \nabla^{2} c_{v} .
$$

For simulating the growth of a void, a 3D model with $128 \times 128 \times 128$ uniform grids were established to solve our phase-field model by using the finite difference algorithm [26]. With the void is spherical shaped, a three-dimensional periodic boundary condition is applied to the simulation domain.

The target of our phase-field simulation is to demonstrate the kinetic of void growth under different doses, which is based on phase-field model developed by Li [24] and Chang [25]. Thus, most of our phase-field model parameters follow these previous studies, where simulations are performed at a temperature of $600 \mathrm{~K}$. The parameters used in the simulations are non-dimensional. The gradient energy coefficients, $\lambda_{i}$ and $\lambda_{v}$, for both vacancies and interstitials are assumed to be both 1.0 at $600 \mathrm{~K}$ [24]. The interstitial mobility and vacancy mobility are 13.46 and 1.0 at $600 \mathrm{~K}$, respectively [24]. The sink bias is 1.1, is adopted in our simulations [25]. Furthermore, the positive constant in chemical/bulk energy density is assumed to be the same, whose value equals to 1.0 [26]. It is challenging to quantify the sink strengths or defect recombination coefficients, phenomenological parameters are adopted to describe the defects sink and recombination process [25]. Usually, the phenomenological parameters of the sink absorption rate or the recombination rate are three orders of magnitude lower than defect generation rate $(G)$ [25]. Thus, with defect generation rate equals to 1.0 and sink dominant assumption, $R_{i v}=0.001$ and $S_{v}=0.2$ are used in the following simulation.

The dose dependence of irradiation-induced swelling by phase-field simulation is shown in Figure 4, where the simulation step can be represented as the evolution time of the void growth or the irradiation dose, the volume fraction of the void can be represented as the radiation-induced swelling. The dose dependence of swelling can be well fitted using a power of 1.5, which is in accordance with our model in Equation (9). We agree that many experimental data [18] show the linear irradiation dose dependence of void swelling at high dose, where swelling rate is about $1 \% / \mathrm{dpa}$. For these experimental data, our model shows better fitting results, which can be found in Figures S2-S4 of Supplementary Materials.

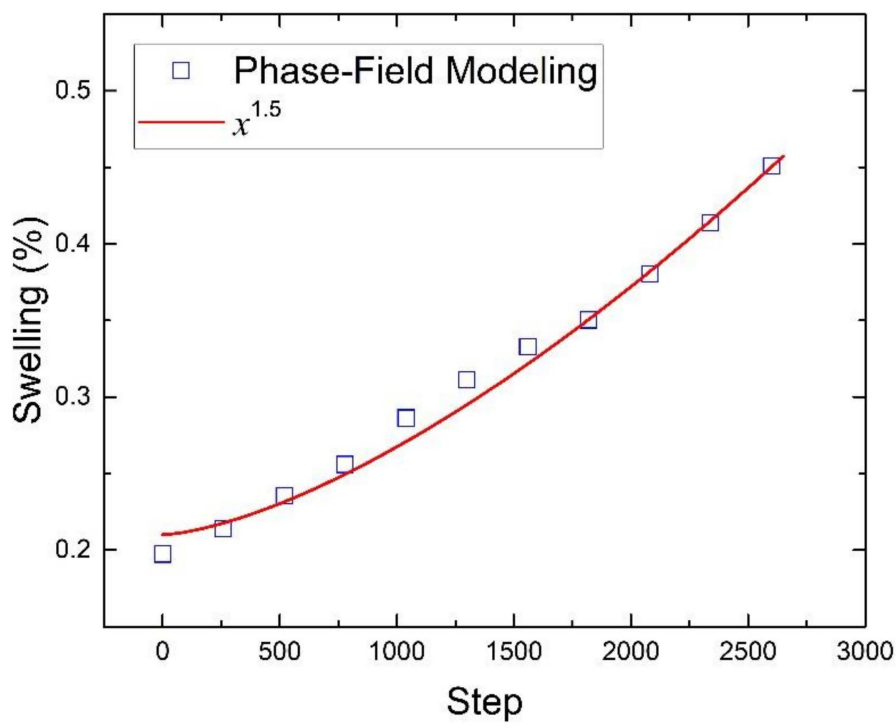

Figure 4. Volume fraction evolution of 3D void under irradiation by phase-field simulation.

Previous works $[1,5,6,13,14,21]$ have demonstrated that volumetric swelling can be influenced by different factors, including the properties of materials such as compositions and their atomic weight, crystal structures, grain boundaries and interfaces, atomic bonds, solute segregation and precipitation, the diffusivity of defects, and sink strength. Meanwhile, irradiation conditions such as irradiation temperature, dose and dose rate also play 
critical roles in irradiation-induced swelling [27,28]. Based on our model, Equation (9) shows irradiation-induced swelling governed by $\alpha, c$, and $\Delta_{d p a}$. The parameter $\alpha$ contains different intrinsic properties of materials and determines how fast the swelling grows. A higher $\alpha$ means faster cavity growth. The value of $c$ is also a material property and relates to the nucleation stage or incubation period for void evolution, which is governed by the threshold dose $\Delta_{0}$ due to the swelling incubation determined by cavity nucleation process. Therefore, a larger $\Delta_{0}$ indicates a longer nucleation/incubation time, suggesting better swelling resistance. The value of $\Delta_{0}$ is important for the development of new materials with improved swelling resistance since the extension of the incubation period is the only meaningful solution for designing swelling-resistant materials up to high doses. The overall swelling of materials relies on both $\Delta_{0}$ and $\alpha$ since the dose-dependent swelling includes an initial nucleation/incubation period, followed by a growth stage [29,30]. In this work, our model describes swelling from different irradiation conditions at the same temperature. Since material properties change with temperatures, temperatures may indirectly affect the swelling through parameters $\alpha$ and $c$. As shown in Table 2, embryo dissolution increases with temperature, indicating that more vacancies for the embryo are needed to grow into a void. Therefore, parameter $c$ increases with temperature. Meanwhile, temperature influences parameter $\alpha$ by diffusion coefficients $D_{i}$ and $D_{v}$, which are in the forms of $D_{i} \sim e^{-E_{m}^{i} / k T}$ and $D_{v} \sim e^{-E_{m}^{v} / k T}$. Thus, Equation (9) is proportional to $\left(b e^{-E_{m}^{v} / T}-e^{-E_{m}^{i} / T}\right)^{1.5}$ where $b$ equals 1.1 as mentioned above, and migration energy for interstitial and vacancy of iron are obtained from previous studies [31,32], $E_{m}^{i}=0.3 \mathrm{eV}, E_{m}^{v}=0.7 \mathrm{eV}$. Parameter $\left(b e^{-E_{m}^{v} / T}-e^{-E_{m}^{i} / T}\right)^{1.5}$ increases with temperature, as plotted in Figure $\mathrm{S} 1$ of the Supplementary Materials. Therefore, parameter $\alpha$ increases with temperature, which is consistent with the tendency in Table 2.

As the point defect absorption by voids and dislocation loops, the total sink absorption rates, $S_{i}$ and $S_{v}$, may not be constant during the irradiation process [33]. Considering the assumption that $S_{i}$ and $S_{v}$ are constant, Equation (9) may be appliable in limited conditions such as the sink dominant case and an elevated temperature within the non-saturation swelling regime. On the one hand, the genuine alloys used in reactors are usually complex metallic alloys, which contains many types of sinks for irradiation-resistance purpose, where sink efficiency is really large. Thus, the changes in sink absorption rates $S_{i}$ and $S_{v}$ are negligible, which can be assumed to be constant. Therefore, we have supposed the sink dominant case for the derivation from Equation (5) to Equation (6). On the other hand, in our model, no saturation of void swelling occurs in materials under irradiation, which is in a good agreement with most experiments. In principle, when voids are large enough under high-dose irradiation, the void surfaces may become the predominant sink for all types of radiation defects. The unbiased sink (voids) will cause biased parameter $(b)$ in parameter $\alpha$, $\alpha=\frac{4 \pi \rho_{N}}{3}\left[2 \Omega \frac{D_{v} b-D_{i}}{\eta S_{i}}\right]^{\frac{3}{2}}$ decrease. Thus, the growth rate will become smaller and smaller, finally reaching zero, in other words, swelling saturation happens. In addition, the density of the network dislocation may decrease at high doses [34]. In these cases, void swelling may saturate at high irradiation doses. However, most experiments show no saturation of void swelling under a given irradiation dose. Particularly, only under certain conditions was found saturation of swelling $[6,20]$. For example, electron irradiation of stainless-steel samples that are too thin leads to an apparent saturation of swelling, which is actually an artifact of the surface impact [6]. In contrast, irradiation leads to steady swelling in bulk samples without saturation [6]. Indeed, Experimental evidence for a high-dose swelling saturation regime is very limited. It came as a big surprise when radiation-induced void swelling was discovered with no indication of a saturation [35]. To validate our model, we have chosen the experimental data that mostly show no saturation of void swelling under irradiation, where the sink absorption rate bias $(b)$ is constant in our model. Accordingly, the material parameters $(\alpha$ and $c$ ) are assumed constant in our model. Therefore, we can only apply the model to swelling in the non-saturation regime, a changing parameter $b$ 
may make our model applicable in the saturation regime of void swelling. Overall, our model-based fittings and extrapolations are in good agreement with available experimental data, suggesting that our introduced concept of supposing the total absorption rate constant successfully captures the essential features.

The understanding and prediction of void swelling is a major long-standing issue facing the development of novel nuclear materials. However, despite decades of experimental and theoretical efforts, this is still the utmost concern for future nuclear reactors. Indeed, irradiation-induced swelling spans multiple time and length scales, the volume swelling of materials is a very complicated process, the modeling of which need to consider a number of factors such as defect production, cluster formation, defect diffusion, sink strength and its bias, interaction between defects/defect clusters with various sinks, and the microstructures/properties of materials, etc. Most previous studies focused on studying specific processes in this whole defect evolution. Although considerable insight has been gained for some evolution stages under specific conditions, an overall understanding of the problem is lacking, especially for the genuine alloys that are used in reactors, which are usually complex metallic alloys. This poses a formidable obstacle for the design of irradiation-resistant structural materials. Additionally, the electron irradiated alloys data for swelling investigation is limited and parameter $\alpha, \frac{4 \pi \rho_{N}}{3}\left[2 \Omega \frac{D_{v} S_{i}-D_{i} S_{v}}{\eta S_{i} S_{v}}\right]^{\frac{3}{2}}$, is governed by intrinsic properties, such as defect diffusion coefficients and the absorption rate of intrinsic defect sinks, including the influence of temperature, pressure, and cold-work conditions on these properties. Thus, a detailed discussion about how material parameters and irradiation conditions affect parameter $\alpha$ and $c$ is really difficult to achieve. Therefore, we just validated our model by extrapolating to high-dose irradiation data and phase-field simulation in another way.

\section{Conclusions}

Considering the main characteristics of defect evolution under energetic electron irradiation, we construct a model for dose dependence of the void swelling in electronirradiated alloys, which is in good agreement with most experimental data. Based on this model, an extrapolation from low-dose to high-dose irradiation-induced swelling is used for model validation. A phase-field simulation is also developed to confirm the dose dependence of the irradiated-induced swelling, which is in accordance with our derived relation between the swelling and irradiation dose. Therefore, this work provides a model for dose dependence of the void swelling in electron-irradiated alloys and a practical solution to evaluate the high-dose swelling effects of the structural materials in advanced nuclear energy systems. Furthermore, by separating material parameters and irradiation conditions in our model, we can study how material parameters affect void swelling and how irradiation dose independently affects void swelling. This provides the basis for void swelling research by neutron irradiation and can play an important guiding role in developing void swelling model for neutron irradiation.

Supplementary Materials: The following supporting information can be downloaded at: https:/ www. mdpi.com/article/10.3390/met12020244/s1. Figure S1: The relation between parameter $\left(b e^{-E_{m}^{v} / T}-e^{-E_{m}^{i} / T}\right)^{1.5}$ and temperature; Figure S2: AISI316 irradiated by neutrons at different temperatures [18]; Figure S3: HT9 and T91 irradiated by $5 \mathrm{MeV} \mathrm{Fe}^{++}$at $460{ }^{\circ} \mathrm{C}$ [36]; Figure S4: $\mathrm{Fe}-20 \mathrm{Ni}-\mathrm{xCr}$ and $\mathrm{Fe}-35 \mathrm{Ni}-\mathrm{xCr}$ irradiated by neutrons at $538^{\circ} \mathrm{C}[18]$.

Author Contributions: Conceptualization, S.Z. and C.W.; Data curation, H.L., J.X., Y.S., J.H. and Z.G.; Funding acquisition, S.Z. and Y.W.; Investigation, W.G. and S.Z.; Supervision, S.Z. and C.W.; Writing—original draft, W.G.; Writing—review and editing, W.G., S.Z., C.W. and Y.W. All authors have read and agreed to the published version of the manuscript.

Funding: This work was supported by National Science Foundation of China (Grant No. 11935004 and Grant No. 12192280). S. Zhao was support by City University of Hong Kong (Grant No. 9610425). 


\section{Institutional Review Board Statement: Not applicable.}

Informed Consent Statement: Not applicable.

Data Availability Statement: The data that supports the findings of this study are available within the article and its supplementary material.

Acknowledgments: The authors thank Steven Zinkle for valuable discussions and suggestions.

Conflicts of Interest: The authors declare no conflict of interest.

\section{References}

1. Zinkle, S.J. Radiation-Induced Effects on Microstructure. Compr. Nucl. Mater. 2020, 1, 65-98. [CrossRef]

2. Mattas, R.; Garner, F.; Grossbeck, M.; Maziasz, P.; Odette, G.; Stoller, R. The impact of swelling on fusion reactor first wall lifetime. J. Nucl. Mater. 1984, 122, 230-235. [CrossRef]

3. Cawthorne, C.; Fulton, E.J. Voids in Irradiated Stainless Steel. Nature 1967, 216, 575-576. [CrossRef]

4. Was, G.S. Fundamentals of Radiation Materials Science: Metals and Alloys; Springer Science \& Business Media: Berlin/Heidelberg, Germany, 2007.

5. Was, G.S. Irradiation-Induced Voids and Bubbles. In Fundamentals of Radiation Materials Science; Springer: Singapore, 2017; pp. 379-484.

6. Garner, F.A.; Thomas, L.E. Production of Voids in Stainless Steel by High-Voltage Electrons; In Proceedings of the ASTM Special Technical Publication; ASTM International: Conshohocken, PA, USA, 1972; pp. 303-323.

7. Han, X.; Tanaka, T.; Kojima, N.; Ohshita, Y.; Yamaguchi, M.; Sato, S. Growth orientation dependent photoluminescence of GaAsN alloys. Appl. Phys. Lett. 2012, 100, 32108. [CrossRef]

8. Bufford, D.C.; Abdeljawad, F.F.; Foiles, S.M.; Hattar, K. Unraveling irradiation induced grain growth with in situ transmission electron microscopy and coordinated modeling. Appl. Phys. Lett. 2015, 107, 191901. [CrossRef]

9. Sato, K.; Yasuda, H. Fluctuation of long-range order in Co-Pt alloy nanoparticles revealed by time-resolved electron microscopy. Appl. Phys. Lett. 2017, 110, 153101. [CrossRef]

10. Knez, D.; Schnedlitz, M.; Lasserus, M.; Hauser, A.W.; Ernst, W.E.; Hofer, F.; Kothleitner, G. The impact of swift electrons on the segregation of Ni-Au nanoalloys. Appl. Phys. Lett. 2019, 115, 123103. [CrossRef]

11. Pavelescu, E.-M.; Ligor, O.; Occena, J.; Ticoş, C.; Matei, A.; Gavrilă, R.L.; Yamane, K.; Wakahara, A.; Goldman, R.S. Influence of electron irradiation and rapid thermal annealing on photoluminescence from GaAsNBi alloys. Appl. Phys. Lett. 2020, 117, 142106. [CrossRef]

12. Was, G.S. Emulating Neutron Irradiation Effects with Ions. Fundam. Radiat. Mater. Sci. 2017, 631-665. [CrossRef]

13. Was, G. Challenges to the use of ion irradiation for emulating reactor irradiation. J. Mater. Res. 2015, 30, 1158-1182. [CrossRef]

14. Hishinuma, A.; Katano, Y.; Shiraishi, K. Dose and Temperature Dependence of Void Swelling in Electron Irradiated Stainless Steel. J. Nucl. Sci. Technol. 1977, 14, 723-730. [CrossRef]

15. Norgett, M.; Robinson, M.; Torrens, I. A proposed method of calculating displacement dose rates. Nucl. Eng. Des. 1975, 33, 50-54 [CrossRef]

16. Was, G.S. Radiation-Enhanced Diffusion and Defect Reaction Rate Theory. Fundam. Radiat. Mater. Sci. 2017, 16, 207-252. [CrossRef]

17. Krsjak, V.; Shen, T.; Degmova, J.; Sojak, S.; Korpas, E.; Noga, P.; Egger, W.; Li, B.; Slugen, V.; Garner, F.A. On the helium bubble swelling in nano-oxide dispersion-strengthened steels. J. Mater. Sci. Technol. 2022, 105, 172-181. [CrossRef]

18. Garner, F.A.; Toloczko, M.B.; Sencer, B.H. Comparison of swelling and irradiation creep behavior of fcc-austenitic and bccferritic/martensitic alloys at high neutron exposure. J. Nucl. Mater. 2000, 276, 123-142. [CrossRef]

19. Garner, F.A. Radiation Damage in Austenitic Steels. In Comprehensive Nuclear Materials; Konings, R.J.M., Stoller, R.E., Eds.; Elsevier: Amsterdam, The Netherlands, 2012; Volume 4, pp. 33-95, ISBN 9780080560335.

20. Norris, D. The use of the high voltage electron microscope to simulate fast neutron-induced void swelling in metals. J. Nucl. Mater. 1971, 40, 66-76. [CrossRef]

21. Makin, M.; Walters, G.; Foreman, A. The void swelling behaviour of electron irradiated type 316 austenitic steel. J. Nucl. Mater. 1980, 95, 155-170. [CrossRef]

22. Gilbon, D.; Rivera, C. Behaviour of different ferritic steels under ion, electron and fast neutron irradiation. J. Nucl. Mater. 1988, 155-157, 1268-1273. [CrossRef]

23. Singh, B.; Horsewell, A.; Gelles, D.; Garner, F. Void swelling in copper and copper alloys irradiated with fission neutrons. J. Nucl. Mater. 1992, 191-194, 1172-1176. [CrossRef]

24. Li, Y.; Hu, S.; Sun, X.; Gao, F.; Henager, C.H.; Khaleel, M. Phase-field modeling of void evolution and swelling in materials under irradiation. Sci. China Phys. Mech. Astron. 2011, 54, 856-865. [CrossRef]

25. Chang, K.; Lee, G.-G.; Kwon, J. A phase-field modeling of void swelling in the Austenitic stainless steel. Radiat. Eff. Defects Solids 2016, 171, 242-251. [CrossRef]

26. Biner, S.B. Solving Phase-Field Models with Finite Difference Algorithms. In Programming Phase-Field Modeling; Springer International Publishing: Singapore, 2017; pp. 17-97. 
27. Okita, T.; Sato, T.; Sekimura, N.; Iwai, T.; Garner, F. The synergistic influence of temperature and displacement rate on microstructural evolution of ion-irradiated Fe-15Cr-16Ni model austenitic alloy. J. Nucl. Mater. 2007, 367-370, 930-934. [CrossRef]

28. Okita, T.; Wolfer, W. A critical test of the classical rate theory for void swelling. J. Nucl. Mater. 2004, 327, 130-139. [CrossRef]

29. Wolfer, W. Advances in void swelling and helium bubble physics. J. Nucl. Mater. 1984, 122, 367-378. [CrossRef]

30. Russell, K. Nucleation of voids in irradiated metals. Acta Met. 1971, 19, 753-758. [CrossRef]

31. Fu, C.C.; Willaime, F.; Ordejón, P. Stability and mobility of mono-and Di-interstitials in $\alpha$-Fe. Phys. Rev. Lett. $2004,92,175503$. [CrossRef] [PubMed]

32. Hashimoto, N.; Sakuraya, S.; Tanimoto, J.; Ohnuki, S. Effect of impurities on vacancy migration energy in Fe-based alloys. J. Nucl. Mater. 2014, 445, 224-226. [CrossRef]

33. Kiritani, M.; Yoshida, N.; Takata, H.; Maehara, Y. Growth of Interstitial Type Dislocation Loops and Vacancy Mobility in Electron Irradiated Metals. J. Phys. Soc. Jpn. 1975, 38, 1677-1686. [CrossRef]

34. Liu, X.; Miao, Y.; Li, M.; Kirk, M.A.; Maloy, S.A.; Stubbins, J.F. Ion-irradiation-induced microstructural modifications in ferritic/martensitic steel T91. J. Nucl. Mater. 2017, 490, 305-316. [CrossRef]

35. Wolfer, W.G. Fundamental Properties of Defects in Metals. In Comprehensive Nuclear Materials; Elsevier: Amsterdam, The Netherlands, 2020; pp. 1-49.

36. Getto, E.; Sun, K.; Monterrosa, A.; Jiao, Z.; Hackett, M.; Was, G. Void swelling and microstructure evolution at very high damage level in self-ion irradiated ferritic-martensitic steels. J. Nucl. Mater. 2016, 480, 159-176. [CrossRef] 